\title{
Editorial
}

\section{Fault Detection, Isolation, and Prognosis for Complex System}

\author{
Chunhui Zhao $\mathbb{D}^{1},{ }^{1}$ Furong Gao, ${ }^{2}$ Yuan Yao, ${ }^{3}$ Zhixing Cao, ${ }^{4}$ and Yongji Fu ${ }^{5}$ \\ ${ }^{1}$ State Key Laboratory of Industrial Control Technology, College of Control Science and Engineering, \\ Zhejiang University, Hangzhou 310027, China \\ ${ }^{2}$ Hong Kong University of Science and Technology, Kowloon, Hong Kong \\ ${ }^{3}$ National Tsing Hua University, Hsinchu, Taiwan \\ ${ }^{4}$ Harvard University, Cambridge, MA, USA \\ ${ }^{5}$ Hill-Rom, Batesville, IN, USA
}

Correspondence should be addressed to Chunhui Zhao; chhzhao@zju.edu.cn

Received 12 December 2017; Accepted 12 December 2017; Published 23 January 2018

Copyright (c) 2018 Chunhui Zhao et al. This is an open access article distributed under the Creative Commons Attribution License, which permits unrestricted use, distribution, and reproduction in any medium, provided the original work is properly cited.

A complex system can be thought of as multiple interdependent working subsystems. With the increasing level of complexity, it may present more frequent unstable operation statuses. Once system faults have occurred, they can cause unrecoverable losses and unacceptable environmental pollution and so forth. It thus demands more effective and efficient techniques to monitor operation status, detect the occurrence and propagation of faults, and enable suitable decision making, before such anomalies result in great damage. As one of the most active research areas over the last few decades, fault diagnosis including detection, isolation, and prognosis has been of importance and necessary for improving the economy and safety of a complex system, ranging from industrial processes, such as steel production, papermaking, car manufacturing, and mineral processing, to biological processes and so forth.

In the era of big data of process industries, new challenge emerges for fault diagnosis with amount of data grown exponentially. In particular, there are many uncertainties in the system which shows the complexity of characteristics, including multimode and dynamics, multilevel and multiscale, nonlinearities, and strong coupling effects amongst the variables. This special issue aims to bring together state-ofthe-art research contributions on fault diagnosis methods and their different applications, as well as future trends. Potential topics of this special issue include fault diagnosis problems for batch processes, fault prognosis for industrial processes, fault self-recovery/self-healing control, product quality monitoring and prediction, process performance assessment, incipient fault detection and diagnosis, fault classification and discrimination, data-driven approaches and knowledge-based approaches, and intelligence-based supervisory control. We received a total of 23 submissions, and after two rounds of rigorous review, 9 papers were accepted.

In the paper "Tooth Fracture Detection in Spiral Bevel Gears System by Harmonic Response Based on Finite Element Method," Y. Chen et al. establish a three-dimensional model and finite element model of the Gleason spiral bevel gear pair. The model considers the effect of tooth root fracture on the system due to fatigue. Finite element method is used to compute the mesh generation, set the boundary condition, and carry out the dynamic load. The harmonic response spectrums of the base under tooth fracture are calculated and the influences of main parameters on monitoring failure are investigated as well.

In the paper "Modeling of Complex Life Cycle Prediction Based on Cell Division," F. Zhang et al. propose to establish a life prediction model by studying the regularity of the cell life in the natural biological evolution. It has moderate complexity which can effectively simulate the state of cell division and does not need a large amount of data in comparison with the conventional life prediction methods. By controlling the concentration of some key factors in cell division, the effect on the normal life of a cell can be expressed intuitively.

In the paper "Degradation Data-Driven Remaining Useful Life Estimation in the Absence of Prior Degradation Knowledge," Y. Yu et al. propose a fusion algorithm to combine the excellent modeling ability of Bayesian updating 
method for the multilevel data and the prominent estimation ability of ECM algorithm for incomplete data. Residual life distributions and posterior distributions are first calculated through the Bayesian updating method based on random initial a priori distributions. Then the a priori distributions are revised and improved for future predictions by the ECM algorithm.

In the paper "Parameter Selection Method for Support Vector Regression Based on Adaptive Fusion of the Mixed Kernel Function," H. Wang and D. Xu use a 5th-degree cubature Kalman filter to estimate the parameters. In this way, they realize the adaptive selection of mixed kernel function weighted coefficients, the kernel parameters, and the regression parameters. Compared with a single kernel function, unscented Kalman filter (UKF) support vector regression algorithms, and genetic algorithms, the decision regression function obtained by the proposed method has better generalization ability and higher prediction accuracy.

In the paper "Time-Varying Fault Diagnosis for Asynchronous Multisensor Systems Based on Augmented IMM and Strong Tracking Filtering," Y. Hu et al. propose a fault detection, isolation, and estimation approach based on Interactive Multimodel (IMM) fusion filtering and Strong Tracking Filtering (STF) for asynchronous multisensors dynamic systems. Time-varying fault is considered and a candidate fault model is built by augmenting the unknown fault amplitude directly into the system state for each kind of possible fault mode. Asynchronous IMM fusion filtering is performed to the multiple model sets to detect and isolate the fault based on model probabilities to avoid the dilemma of traditional IMM-based approaches.

In the paper "A Bayesian Approach to Control Loop Performance Diagnosis Incorporating Background Knowledge of Response Information," S. Zhou and Y. Wang incorporate background knowledge into Bayesian inference to isolate the problem source degrading the control loop performance. In an effort to reduce dependence on the amount of historical data available, they consider a general kind of background knowledge, known as response information, and translate it to constraints on the underlying probability distributions. In this way, the dimensionality of the observation space is reduced and thus the diagnosis can be more reliable.

In the paper "WOS-ELM-Based Double Redundancy Fault Diagnosis and Reconstruction for Aeroengine Sensor," Z. Zhao et al. propose a double redundancy diagnosis approach based on Weighted Online Sequential Extreme Learning Machine (WOS-ELM) in order to diagnose sensor fault of aeroengine more quickly and accurately. It assigns different weights to old and new data and implements weighted dealing with the input data to get more precise training models. The WOS-ELM-based double redundancy fault diagnosis and reconstruction approach contains two diagnosis models which can real-timely detect the hard fault and soft fault much earlier.

In the paper "Development of Fault Identification System for Electric Servo Actuators of Multilink Manipulators Using Logic-Dynamic Approach," V. Filaretov et al. present synthesis method of faults identification systems based on logicdynamic approach for electric servo actuators of multilink manipulators which are described by nonlinear equations with significantly changing coefficients. An advantage of this approach is that it allows studying systems with nonsmooth nonlinearities by linear methods only.

In the paper "Fault Diagnosis of Nonlinear Uncertain Systems with Triangular Form," Q. Ding et al. propose a fault diagnosis approach for a class of nonlinear uncertain systems with triangular form based on the extended state observer (ESO) of the active disturbance rejection controller and linearization of dynamic compensation. This method is reported to be of utility and simple in construction and parameter tuning.

\section{Acknowledgments}

The guest editorial team would like to thank authors of all the papers submitted to this special issue. Given the space limitations, a number of high quality contributions could not be accommodated. The editors also wish to thank the anonymous reviewers, some of whom helped with multiple review assignments. We hope that you will enjoy reading this special issue devoted to this exciting and fast-evolving field as much as we have done.
Chunhui Zhao Furong Gao Yuan Yao Zhixing Cao Yongji Fu 


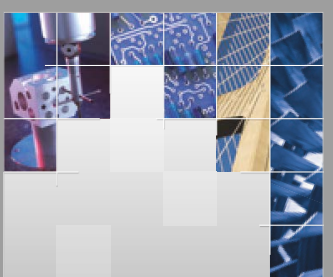

\section{Enfincering}
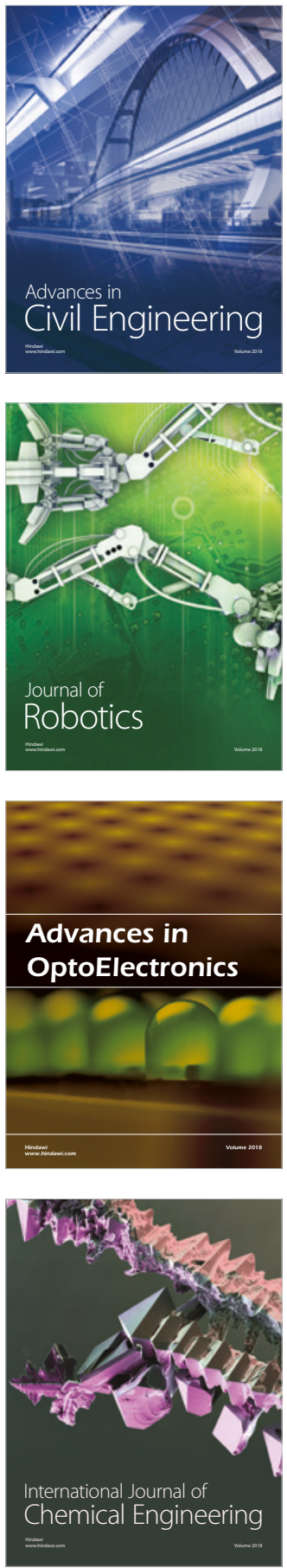

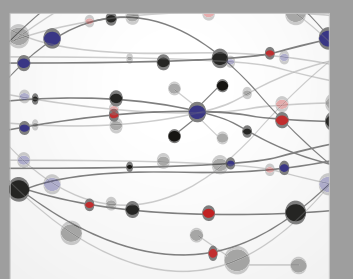

\section{Rotating \\ Machinery}

The Scientific World Journal

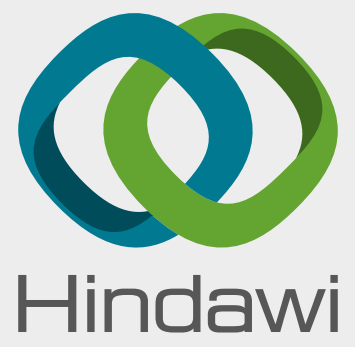

Submit your manuscripts at

www.hindawi.com
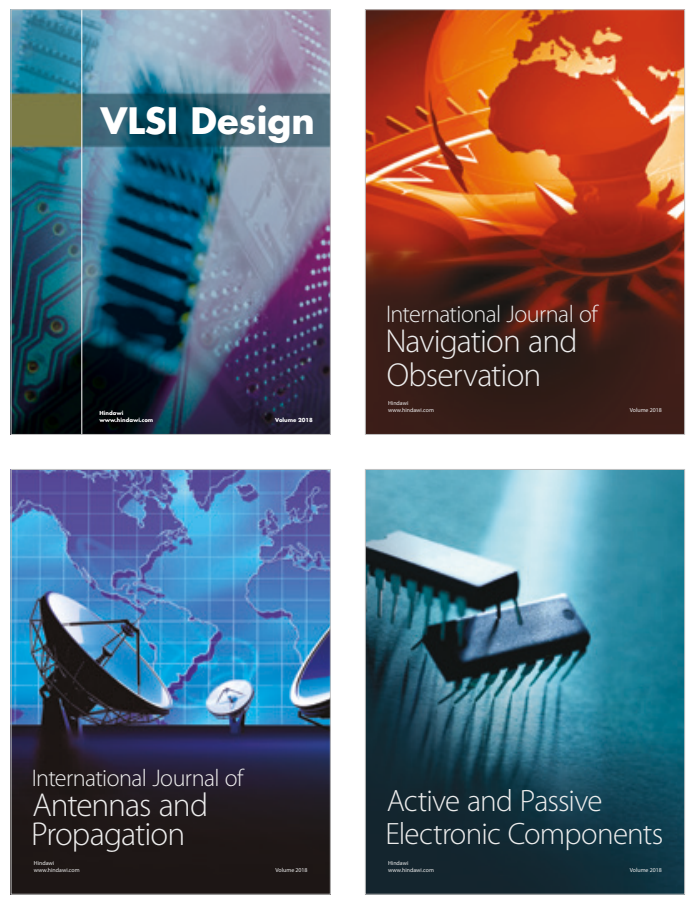
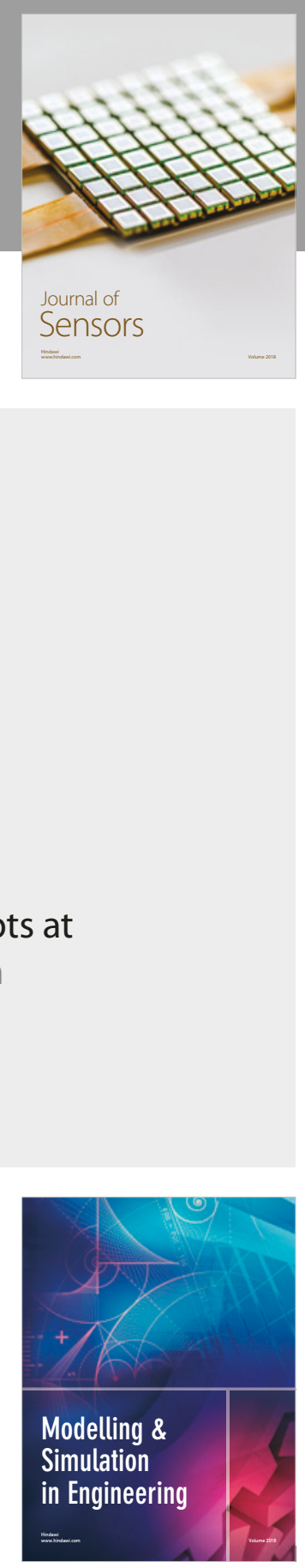

\section{Advances \\ Multimedia}
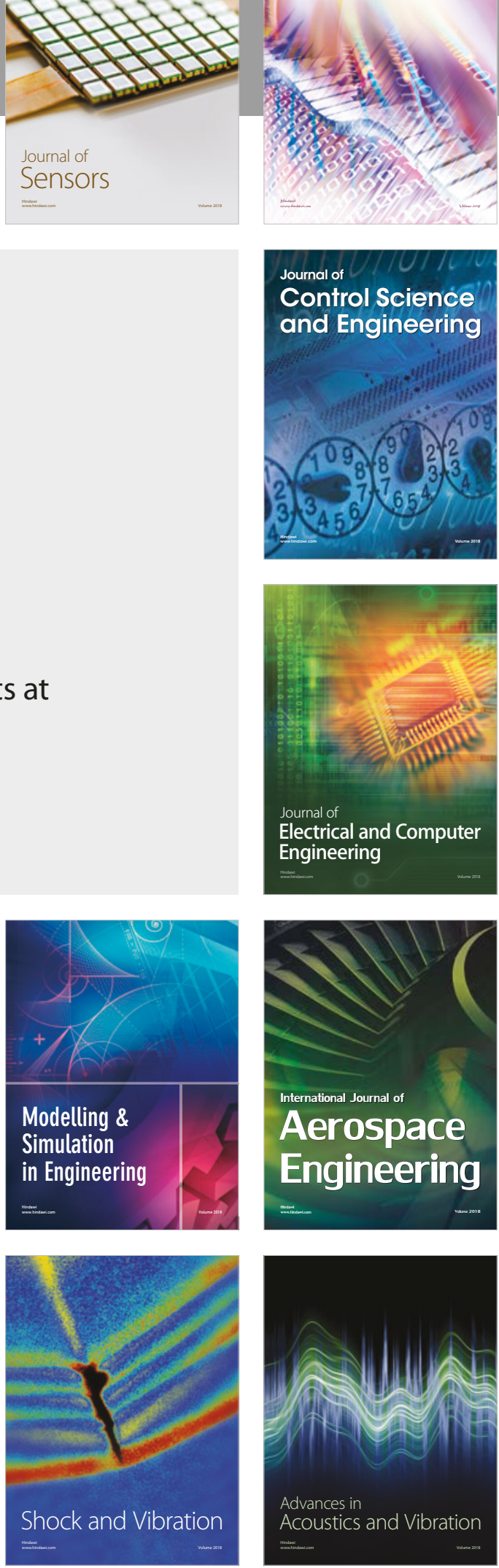